\title{
Predictive Accuracy of MCI Subtypes for Alzheimer's Disease and Vascular Dementia in Subjects with Mild Cognitive Impairment: A 2-Year Follow-Up Study
}

\author{
S.M.C. Rasquin ${ }^{a} \quad$ J. Lodder ${ }^{b}$ P.J. Visser ${ }^{a} \quad$ R. Lousberg ${ }^{a}$ F.R.J. Verhey ${ }^{a}$ \\ Research Institute Brain and Behavior, a Department of Psychiatry and Neuropsychology, and bepartment of \\ Neurology, University of Maastricht/University Hospital Maastricht, Maastricht, The Netherlands
}

\section{Key Words}

Mild cognitive impairment • Predementia stage •

Vascular dementia · Alzheimer's dementia • Diagnostic criteria

\begin{abstract}
Aim: The aim of this study was to investigate the prognostic accuracy of different subtypes of mild cognitive impairment (MCl): amnestic $\mathrm{MCl}$, multiple domain $\mathrm{MCl}$, and single non-memory domain $\mathrm{MCl}$, for the development of Alzheimer's dementia (AD) and vascular dementia (VaD). Patients: Nondemented patients from a memory clinic cohort ( $n=118$ ), and a stroke cohort ( $n=80$, older than 55 years and with a cognitive impairment). Results: 'Multiple domain $\mathrm{MCl}^{\prime}$ had the highest sensitivity for both $\mathrm{AD}(80.8 \%)$ and $\mathrm{VaD}(100 \%)$, and 'amnestic $\mathrm{MCl}^{\prime}$ had the highest specificity $(85.9 \%$ for $A D, 100 \%$ for $\mathrm{VaD})$. The positive predictive value was low for all subtypes $(0.0-32.7 \%)$, whereas the negative predictive value was high (72.8-100\%). Discussion: The subtype 'multiple domain $\mathrm{MCl}^{\prime}$ has high sensitivity in identifying people at risk for developing $A D$ or $\mathrm{VaD}$. The predictive accuracy of the $\mathrm{MCl}$ subtypes was similar for both $\mathrm{AD}$ and $\mathrm{VaD}$.
\end{abstract}

Copyright @ 2005 S. Karger AG, Basel

\section{Introduction}

Mild cognitive impairment (MCI) is a term referring to persons who do not fulfill criteria for dementia, but who exhibit some form of cognitive impairment. The most common causes of MCI are age-related cognitive impairment, the predementia stages of Alzheimer's disease (AD), and vascular dementia (VaD). It has recently been proposed that specific subtypes of MCI may be useful to distinguish between these three conditions [1]. For example, subjects with isolated memory impairment (amnestic $\mathrm{MCI}$ ) may be at increased risk of developing AD, while subjects with impairments in multiple cognitive domains (multiple domain MCI) may be at increased risk of developing $\mathrm{VaD}$, and patients with a single non-memory domain impairment may be at increased risk of developing frontotemporal lobe dementia [1]. This proposal has not yet been tested in a clinical setting, but a recent population-based study failed to find support for the proposal [2].

The aim of the present study is to investigate whether 'amnestic MCI', 'multiple domain MCI', and 'single nonmemory domain MCI' are diagnostic entities for the predementia stage of $\mathrm{AD}$ and $\mathrm{VaD}$ in two separate cohorts (memory clinic and stroke cohort) of subjects with MCI.

\begin{tabular}{ll}
\hline KARGER & ( ) 2005 S. Karger AG, Basel \\
Fax +4161306 1234 $34-8008 / 05 / 0193-0113 \$ 22.00 / 0$ \\
$\begin{array}{l}\text { E-Mail karger@karger.ch } \\
\text { www.karger.com }\end{array}$ & $\begin{array}{l}\text { Accessible online at: } \\
\text { www.karger.com/dem }\end{array}$
\end{tabular}

Prof. Dr. F.R.J. Verhey

Department Psychiatry and Neuropsychology

PO Box 5800, NL-6202 MD Maastricht (The Netherlands)

Tel. +31 43 3877537, Fax +31433875444

E-Mail F.Verhey@np.unimaas.nl 
Table 1. Cognitive domains, tests used and definition of impairment

\begin{tabular}{|c|c|c|}
\hline Domains & Test & Impairment \\
\hline Memory & $\begin{array}{l}\text { AVLT, total words direct recall and } \\
\text { delayed recall }\end{array}$ & score $\leq 10^{\text {th }}$ percentile \\
\hline $\begin{array}{l}\text { Executive } \\
\quad \text { functioning }\end{array}$ & mean interference score SCWT, CST or TMT & score $\leq 10^{\text {th }}$ percentile \\
\hline Calculation & GIT & score $\leq 10^{\text {th }}$ percentile \\
\hline Visuospatial & GIT & score $\leq 10^{\text {th }}$ percentile \\
\hline Orientation & MMSE items 1 and 2 (place, person, time) & score $\leq 8$ \\
\hline Attention & MMSE item 4 (serial 7’s) & score $\leq 4$ \\
\hline Praxis & MMSE item 11 (copying) & score $=0$ \\
\hline Language & $\begin{array}{l}\text { MMSE items } 6,7,8,9,10 \text { (understanding, } \\
\text { expression, writing) }\end{array}$ & score $\leq 6$ \\
\hline Mental speed & SCWT I, CST I and II or TMT I and II & score $\leq 10^{\text {th }}$ percentile \\
\hline
\end{tabular}

AVLT $=$ Auditory Verbal Learning Test SCWT $=$ Stroop Color Word Test CST $=$ Concept Shifting Test; TMT $=$ Trail Making Test $;$ GIT $=$ Groninger Intelligence Test MMSE = Mini Mental State Examination.

Interference score: part III - mean (part I + part II).
As there is controversy about the emphasis of an (isolated) memory impairment in the concept MCI [3-5], we further subdivided the subtype 'multiple domain MCI' into: 'multiple domain MCI with memory impairment included' (memory+) and 'multiple domain MCI with memory impairment excluded' (memory-).

\section{Patients and Methods}

\section{Patients}

Two clinical cohorts of nondemented, cognitively impaired subjects were available for this study: a cohort of patients with memory complaints who contacted the Memory Clinic of the University Hospital Maastricht, and a cohort of first-ever stroke patients from the stroke unit of the same hospital. Patients from the memory clinic cohort were participants of a longitudinal study of mild cognitive impairment, which started in 1990 [6]. The stroke patients were participants of a longitudinal study called CODAS (Cognitive Disorders after Stroke) [7-9].

Inclusion criteria for this study were; age older than 55 years, a cognitive impairment affecting at least 1 out of 9 cognitive domains (see below), no interference with daily activities (based on a questionnaire for the informant), and follow-up longer than 2 years. Exclusion criteria were dementia at baseline (defined according to DSM-IV), an MMSE score $<15$ (to ensure valid neuropsychological testing), and the presence of any somatic or psychiatric disorder that could be related to the cognitive impairment, except a first-ever stroke in the CODAS cohort. Patients from the memory clinic cohort were excluded if they had cerebrovascular disorders, defined by a score on the Hachinski Ischemic Scale of 5 or higher [10]. In the CODAS cohort, patients with a previous stroke or a stroke in the cerebellum or brainstem were excluded.
Patients from the MCI cohort who were excluded because the follow-up period was too short $(n=44)$ were older than the included patients, but MMSE scores, sex ratio and level of education were not different. Patients from the stroke cohort who were excluded because the follow-up period was too short $(n=34)$ were older and had lower baseline MMSE scores than the included patients, but sex ratios and level of education were not different.

\section{Method}

Patients from the longitudinal MCI study were tested at baseline and 24 months later. Patients from the CODAS study were tested at $1,6,12$, and 24 months after stroke. This report used the data from the baseline and 24 months' assessments.

\section{Cognitive Domains}

Table 1 presents the cognitive domains investigated. The neuropsychological tests used have been described extensively in earlier reports $[7,8,11]$.

The choice of the domains studied was based on the criteria for VaD proposed by the National Institute of Neurological Disorders and Strokes - Association Internationale pour la Recherche et l'Enseignement en Neurosciences (NINDS-AIREN) [12]. 'Memory' was evaluated with the Auditory Verbal Learning Test [13]; 'executive functioning' with a combination of the interference score of the Stroop Color Word Test (SCWT) [14] and with the interference score of either the Concept Shifting Test (CST) [15] or the Trail Making Test (TMT) [16]; 'calculation' and 'visuospatial abilities' with subscales of the Groninger Intelligence Scale [17], and 'orientation', 'attention', 'praxis', and 'language' with subtests of the MMSE [18]. In addition to the 8 cognitive domains, we included also tests to assess 'mental speed', as this aspect may be of relevance according to previous studies [7, 19]. 'Mental speed' was evaluated with a combination of the scores of the first part of the SCWT [14] and with the scores on the first and second part of either the CST [15] or the TMT [16]. Both cohorts were administered the same neuropsychological battery with one exception: patients from the memory clinic cohort 
Table 2. Demographic characteristics of the two cohorts

\begin{tabular}{|c|c|c|}
\hline & Memory-clinic cohort & Stroke cohort \\
\hline $\mathrm{n}$ & 118 & 80 \\
\hline Age (mean, SD) & $64.8(7.5)$ & $70.4(7.8)^{*}$ \\
\hline Level of education, $\%$ low & 23.7 & $56.3^{*}$ \\
\hline Sex, $\%$ female & 48.3 & 55.0 \\
\hline MMSE score (mean, SD) & $27.3(2.2)$ & $26.3(2.4)^{*}$ \\
\hline
\end{tabular}

completed the TMT, whereas patients from the CODAS study completed the CST. Because the TMT and CST measure the same cognitive function and are highly correlated it was valid to assess the tests in each single cohort [15]. The performance of both cohorts was compared with that of a normative group from the Maastricht Aging Study [11]. The aim of this study was to investigate factors that predispose to normal cognitive aging in a cohort of healthy elderly volunteers from the southern part of the Netherlands. Normative data from this study were stratified according to age, sex, and educational level. Individual test scores were converted to standard Z scores, in order to define impairment in the cognitive functions, using the means and standard deviations of the normative data (test score patient - mean norm group)/SD norm group.

\section{Diagnosis of Dementia}

At 24 months, and without knowledge of the baseline data, two experienced clinicians independently evaluated patients regarding a diagnosis of dementia according to the DSM-IV criteria [20], based on available data, such as information about medical history, test performance, structured interview with an informant, and a clinical observation. Interference in daily activities due to cognitive impairments was evaluated by means of questionnaires about daily activities that were completed by the informant [21]. The diagnosis of AD was based on the National Institute for Neurological and Communicative Disorders and Stroke-Alzheimers Disease and Related Disorders Association (NINCDS-ADRDA) criteria and the diagnosis of $\mathrm{VaD}$ was based on the NINDS-AIREN criteria.

If there was a discrepancy in diagnosis a consensus meeting was arranged. If no consensus could be reached the patient was considered not demented. Agreement between the two clinicians was high $(\kappa=0.88 ; \mathrm{p}=0.01)$.

\section{Diagnosis of $M C I$}

MCI was defined if patients had: (1) an impairment in at least 1 of the 9 cognitive domains; (2) no interference in daily activities; (3) no dementia according to the DSM-IV. A cognitive impairment was defined as a score lower than the 10th percentile of the $\mathrm{z}$ scores, based on the norm scores, compared to the norm group (equivalent of 1.28 SD below the norm scores) or an MMSE score lower than $80 \%$ of the maximum score on the relevant scale.

The descriptions proposed by Petersen et al. [1] were used to subclassify MCI. Patients were classified as 'amnestic MCI' if they had a memory impairment, but were performing otherwise well (z score $>1.28$ compared to the norm group) on other indexes of cognitive function. Patients were classified as 'multiple domain MCI' if they had impairments on 2 or more domains. This latter concept corre- sponds with that of Cognitive Impairment No Dementia (CIND) [5, 22, 23]. Moreover we made a subclassification in 'multiple domain MCI memory+' if patients had impairments on 2 or more domains, one of which was memory impairment and 'multiple domain $\mathrm{MCI}$ memory-' if patients had impairments on 2 or more domains but no memory impairment. Patients were classified as 'single non-memory domain MCI' if they had an impairment in a single domain other than memory.

\section{Statistical Analyses}

Descriptive statistics were used for the demographic variables and the number of patients that became demented. Baseline differences between the two groups with regard to the number of cognitive impairments were tested with a chi-square test or $t$ test. The validity and predictive value of MCI subtype as being diagnostic for the development of dementia were determined with sensitivity, specificity, and predictive values. Analyses were performed with the Statistical Package for Social Sciences version 10 (SPSS-10) for Macintosh.

\section{Results}

In total 118 patients from the memory clinic cohort and 80 patients from the stroke cohort were included in this study. Patients from the stroke cohort had a lower educational level, were older, and had lower baseline MMSE score than patients from the memory clinic cohort (table 2).

At baseline compared with patients from the memory clinic cohort, patients from the stroke cohort more often had an impairment in calculation $\left(\chi^{2}=16.3\right.$, d.f. $=1, \mathrm{p}=$ $0.01)$, visuospatial functioning $\left(\chi^{2}=14.3\right.$, d.f. $=1, \mathrm{p}=$ $0.01)$, attention $\left(\chi^{2}=8.8\right.$, d.f. $\left.=1, p=0.01\right)$, language $\left(\chi^{2}=\right.$ 8.7, d.f. $=1, \mathrm{p}=0.01)$, and praxis $\left(\chi^{2}=15.2\right.$, d.f. $=1, \mathrm{p}=$ $0.01)$, but less often an impairment in memory $\left(\chi^{2}=34.4\right.$, d.f. $=1, \mathrm{p}=0.01)$ (fig. 1).

In both cohorts most patients ( 63.5 and $73.8 \%$, respectively) had 'multiple domain MCI' (table 3 ).

In the memory clinic cohort, 26 patients were classified as demented $(22.0 \%)$ at follow-up, all being diagnosed with $\mathrm{AD}$ according to the NINCDS-ADRDA. In the stroke cohort only 5 patients had dementia at followup (6.3\%), all of whom were diagnosed with $\mathrm{VaD}$ according to the NINDS-AIREN criteria.

The sensitivity and specificity of the four MCI subtypes for the diagnosis of either $\mathrm{AD}$ or $\mathrm{VaD} 2$ years later are presented in table 4 . The subtype 'multiple domain MCI' had high sensitivity for the development of both AD and $\mathrm{VaD}$. Specificity rates were highest for the amnestic type in both cohorts. For all MCI subtypes the positive predictive value was low (range $0.0-32.7 \%$ ), but the negative predictive value was higher for all MCI subtypes (range $72.8-100 \%$ ). 
Fig. 1. Percentages of patients with an impairment.

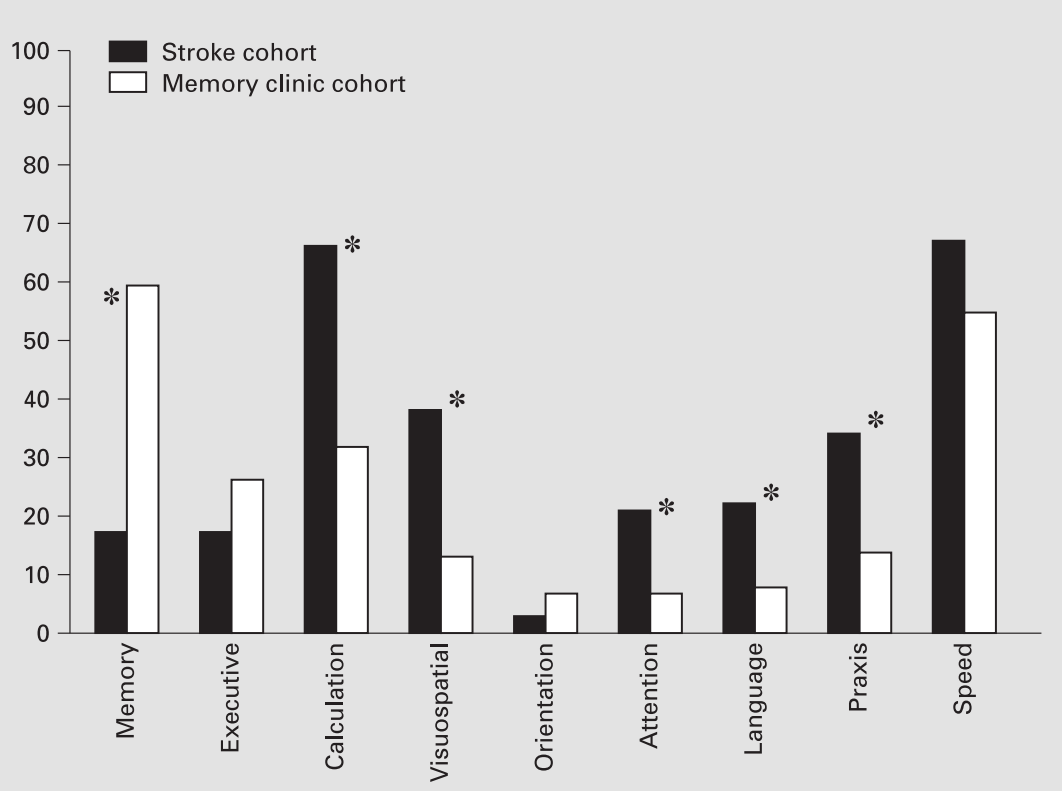

Table 3. Distribution of patients according to different MCI subtypes

\begin{tabular}{llllll}
\hline & Amnestic & \multicolumn{2}{l}{ Multiple domains MCI } & \multirow{2}{*}{$\begin{array}{l}\text { Single } \\
\text { non-memory }\end{array}$} \\
\cline { 3 - 5 } & MCI & all & memory+ & memory- & \\
\hline Memory clinic cohort & $17(14.4 \%)$ & $75(63.5 \%)$ & $52(44.1 \%)$ & $23(19.5 \%)$ & $26(22.0 \%)$ \\
Without dementia at follow-up & 13 & 54 & 35 & 19 & 25 \\
With AD at follow-up & 4 & 21 & 17 & 4 & 1 \\
\hline Stroke cohort & 0 & $59(73.8 \%)$ & $13(16.3 \%)$ & $46(57.5 \%)$ & $21(26.2 \%)$ \\
Without dementia at follow-up & 0 & 54 & 11 & 43 & 21 \\
With VaD at follow-up & 0 & 5 & 2 & 3 & 0 \\
\hline
\end{tabular}

To investigate which individual cognitive domain had the highest predictive value we did a post hoc analysis into the predictive values for the development of either $\mathrm{AD}$ or $\mathrm{VaD}$ of each separate domain. Orientation had the highest positive predictive value for the development of $\mathrm{AD}(\mathrm{PPV}=62.5 \%, \mathrm{CI}=0.29-0.96)$ and memory had the highest negative predictive value for the development of $\mathrm{AD}(\mathrm{NPV}=91.5 \%, \mathrm{CI}=0.84-0.99)$. For the development of $\mathrm{VaD}$, attention had the highest positive predictive value $(\mathrm{PPV}=18.0 \%, \mathrm{CI}=0.00-0.36)$, and both mental speed and executive functioning had the highest negative predictive value $\left(\mathrm{NPV}_{\text {speed }}=100 \% \mathrm{CI}=1.0-1.0\right.$ and $\left.\mathrm{NPV}_{\text {executive }}=100 \%, \mathrm{CI}=1.0-1.0\right)$.

\section{Discussion}

This is the first study to establish the prognostic value of the different MCI subtypes in a clinical population [1]. In the memory clinic cohort, the 'multiple domain MCI' subtype was the most prevalent, and the 'amnestic MCI' subtype the least. This suggests that isolated memory impairment is rare and that the amnestic MCI subtype has limited applicability in clinical practice, unlike 'multiple domain MCI', which would appear to be a more relevant subtype [5]. Indeed, 'amnestic MCI' had a low sensitivity as a prognostic test for the development of $\mathrm{AD}$, whereas 'multiple domain MCI' had a high sensitivity. 
Table 4. Sensitivity and specificity for the four MCI subtypes for the development of either $\mathrm{AD}$ or $\mathrm{VaD}$

\begin{tabular}{lcclc}
\hline & $\begin{array}{l}\text { Sensitivity } \\
(95 \% \mathrm{CI})\end{array}$ & $\begin{array}{l}\text { Specificity } \\
(95 \% \mathrm{CI})\end{array}$ & $\begin{array}{l}\text { PPV } \\
(95 \% \mathrm{CI})\end{array}$ & $\begin{array}{l}\text { NPV } \\
(95 \% \mathrm{CI})\end{array}$ \\
\hline Memory clinic cohort & & & & \\
Amnestic MCI & $15.4 \%$ & $85.9 \%$ & $23.5 \%$ & $78.2 \%$ \\
& $(0.02-0.29)$ & $(0.79-0.93)$ & $(0.03-0.44)$ & $(0.7-0.86)$ \\
Multiple domains all & $80.8 \%$ & $41.3 \%$ & $28.0 \%$ & $88.4 \%$ \\
& $(0.66-0.96)$ & $(0.31-0.51)$ & $(0.18-0.38)$ & $(0.79-0.98)$ \\
Multiple domains MCI & $65.4 \%$ & $61.9 \%$ & $32.7 \%$ & $86.4 \%$ \\
memory+ & $(0.47-0.84)$ & $(0.52-0.72)$ & $(0.20-0.45)$ & $(0.78-0.95)$ \\
Multiple domains MCI & $15.4 \%$ & $79.3 \%$ & $17.3 \%$ & $76.8 \%$ \\
memory- & $(0.02-0.29)$ & $(0.71-0.88)$ & $(0.02-0.33)$ & $(0.68-0.85)$ \\
Single non-memory MCI & $3.8 \%$ & $72.8 \%$ & $3.8 \%$ & $72.8 \%$ \\
& $(0.0-0.11)$ & $(0.64-0.82)$ & $(0.0-0.11)$ & $(0.64-0.82)$ \\
\hline Stroke cohort & & & & \\
Amnestic MCI & $0.0 \%$ & $100 \%$ & $0.0 \%$ & $93.8 \%$ \\
Multiple domains all & $(0.0-0.0)$ & $(1.0-1.0)$ & $(0.0-0.0)$ & $(0.88-0.99)$ \\
& $100 \%$ & $28.0 \%$ & $8.5 \%$ & $100 \%$ \\
Multiple domains MCI & $(1.0-1.0)$ & $(0.18-0.38)$ & $(0.01-0.16)$ & $(1.0-1.0)$ \\
memory+ & $40.0 \%$ & $85.3 \%$ & $15.4 \%$ & $95.5 \%$ \\
Multiple domains MCI & $(0.0-0.83)$ & $(0.77-0.93)$ & $(0.0-0.35)$ & $(0.91-1.0)$ \\
memory- & $60.0 \%$ & $42.7 \%$ & $6.5 \%$ & $94.1 \%$ \\
Single non-memory MCI & $(0.17-1.03)$ & $(0.31-0.54)$ & $(0.0-0.14)$ & $(0.86-1.02)$ \\
& $0.0 \%$ & $73.8 \%$ & $0.0 \%$ & $92.1 \%$ \\
& $(0.0-0.0)$ & $(0.62-0.82)$ & $(0.0-0.0)$ & $(0.84-0.99)$ \\
\hline
\end{tabular}

$\mathrm{CI}=$ Confidence interval.
Other investigators also found $\mathrm{MCI}$ or related concepts such as CIND to be valid with regard to the development of dementia (mostly AD) [5, 22-24]. The low positive predictive value of the MCI subtypes found in our study may be because of the small number of cases of dementia diagnosed and the short follow-up. The results suggest that this subtype classification of MCI is of limited clinical relevance. The negative predictive value of these subtypes was high, the highest being for 'multiple domain MCI'. This indicates that if patients are not diagnosed as 'multiple domain MCI', the chance of them becoming demented is low.

In the stroke cohort 'multiple domain MCI' was the most common subtype and 'amnestic MCI' the least. 'Multiple domain MCI' had the highest sensitivity and 'amnestic MCI' had the highest specificity for the predementia stage of $\mathrm{VaD}$. In their study of stroke patients, Ballard et al. [25] did not find MCI and other related concepts to be useful as indicators of the transition phase to dementia after stroke. Despite of these negative results, nondemented patients with cerebrovascular diseases and with mild cognitive deficits are at increased risk of developing dementia [22, 23, 26-28].

Predictive Accuracy of MCI Subtypes for Dementia
In both cohorts, the 'multiple domain MCI' subtype was the most prevalent. Also others found that the cognitive profile of patients in the predementia stage is characterized by multiple domains impaired, which accords with the concept of CIND [5, 22, 23]. We further divided this concept and found that in the stroke cohort, 'multiple domain MCI memory-' was more than 3 times more prevalent than the 'multiple domain MCI memory+'. These findings suggest that the cognitive profile of patients with cerebrovascular disease differs from that of patients with other disorders. This is in line with the analysis of the individual cognitive domains in which patients from the MCI cohort had more often memory deficits. In combination with our finding that memory deficits had the highest negative predictive value, patients without a memory deficit had thus a lower chance of becoming demented than patients with a memory deficit. In the stroke cohort patients without executive functioning or without mental speed deficits had a lower chance of becoming demented compared to patients with these deficits. Busse et al. [2] failed to find relation between MCI subtypes and the development of dementia, which may be because they used a population-based design in which

Dement Geriatr Cogn Disord 2005;19:113-119 
subjects with neurological disorders were excluded. As a result, subjects who were at higher risk of developing $\mathrm{VaD}$ were underrepresented [2].

This study has some shortcomings. Firstly, patients from the two cohorts visited the hospital for different reasons: patients from the memory cohort had memory complaints whereas patients from the stroke cohort had had a stroke. However, despite these differences in referral, the groups were similar with regard to cognitive functioning (MMSE score at baseline differed only 1 point), and inclusion and exclusion criteria. Secondly, the patients in the stroke cohort were tested four times, and those in the memory clinic cohort only two times, which may have led to differences caused by a learning effect. However, as dementia was primarily diagnosed on the basis of the clinical assessment, we consider this effect to be of limited relevance in our study. Thirdly, we diagnosed MCI and its subtypes based on psychometric definitions and not in combination with a clinical judgment. This is in contrast with the clinical diagnosis of MCI, as described by Petersen et al. [1]. Moreover, we did not take memory complaints into consideration, which means we may have overestimated the number of patients with MCI because other studies have shown that the prevalence of MCI increases when subjective cognitive complaints are not taken into consideration [2, 29]. Fourthly, because few patients were diagnosed with dementia, the power of the study was low, which may be a consequence of the exclusion of people with an MMSE score $<15$. Lastly, the follow-up period was relatively short, which could have led to an underestimation of the positive predictive value of the various subtypes of MCI.

In conclusion, the subtype 'multiple domain MCI' has the highest sensitivity in predicting both $\mathrm{AD}$ and $\mathrm{VaD}$. A high sensitivity in diagnosing patients who are at risk of developing dementia is desirable in the light of intervention possibilities.

We propose that patients who meet the diagnostic criteria of subtype 'multiple domain MCI' are in the predementia stage and have a high risk of going on to develop dementia. However, the low positive predictive value indicates that other markers for the predementia stage are needed.

\section{Acknowledgments}

We would like to thank I. Winkens for the neuropsychological assessment and R. Ponds for his contribution to the diagnosis of dementia.

This study was supported by grants from the Adriana van Rinsum-Ponssen Foundation.

\section{References}

-1 Petersen RC, Doody R, Kurz A, et al: Current concepts in mild cognitive impairment. Arch Neurol 2001:58:1985-1992.

-2 Busse A, Bischkopf J, Riedel-Heller SG, Angermeyer MC: Subclassifications for mild cognitive impairment: Prevalence and predictive validity. Psychol Med 2003;33:1029-1038.

-3 DeCarli C: Mild cognitive impairment: Prevalence, prognosis, aetiology, and treatment. Lancet Neurol 2003;2:15-21.

-4 Petersen RC, Smith GE, Waring SC, et al: Mild cognitive impairment: Clinical characterization and outcome. Arch Neurol 1999;56:303308.

-5 Ritchie K, Touchon J: Mild cognitive impairment: Conceptual basis and current nosological status. Lancet 2000;355:225-228.

-6 Visser PJ, Verhey FRJ, Ponds RWHM, Kester A, Jolles J: Distinction between preclinical Alzheimer's disease and depression. J Am Geriatr Soc 2000;48:479-484.

7 Rasquin SMC, Verhey FRJ, Lousberg R, Winkens I, Lodder J: Vascular cognitive disorders: Memory, mental speed and cognitive flexibility after stroke. J Neurol Sci 2002;203204(C):115-119.
Rasquin SMC, Lodder J, Ponds RWHM, Jolles J, Winkens I, Verhey FRJ: Cognitive functioning after stroke: A one-year follow-up study. Dement Geriatr Cogn Disord 2004;18:138144.

9 Rasquin SMC, Lodder J, Verhey FRJ: Predictors of reversible mild cognitive impairment after stroke: A two year follow-up study. J Neurol Scie 2004, in press.

10 Hachinski VC, Lassen NA, Marshall J: Multiinfarct dementia: A cause of mental deterioration in the elderly. Lancet 1974;ii:207-210.

11 Jolles J, Houx PJ, van Boxtel MPJ, Ponds RWHM: The Maastricht Aging Study: Determinants of Cognitive Aging. Maastricht, Neuropsych Publishers, 1995.

12 Roman GC, et al: Vascular dementia: Diagnostic criteria for research studies. Report of the NINDS-AIREN International Workshop. Neurology 1993:43:250-260.

13 Brand N, Jolles J: Learning and retrieval rate of words presented auditorily and visually. J Gen Psychol 1985;112:201-210.

14 Houx PJ, Jolles J, Vreeling FW: Stroop interference: Aging effects assessed with the Stroop Color-Word Test. Exp Aging Res 1993; 19:204224.
15 Vink M, Jolles $\mathrm{J}$ : A new version of the TrailMaking Test as an information processing task. J Clin Neuropsychol 1985;7:162.

16 Reitan RM: Validity of the Trail Making Test as an indication of organic brain damage. Percept Motor Skills 1985;8:271-276.

17 Luteijn F, van der Ploeg FAE: Manual Groninger Intelligence Test. Lisse, Swets \& Zeitlinger, 1983.

18 Folstein MF, Folstein SE, McHugh PR: 'Minimental state': A practical method for grading the cognitive state of patients for the clinician. J Psychiatr Res 1975;12:189-198.

19 Bowler JV, Hadar U, Wade JP: Cognition in stroke. Acta Neurol Scand 1994;90:424-429.

20 American Psychiatric Association: Diagnostic and Statistical Manual of Mental Disorders, 4 ed. Washington, American Psychiatric Association, 1994.

21 Teunisse S, Derix MM: Measurement of activities of daily living in patients with dementia living at home: Development of a questionnaire (in Dutch). Tijdschr Gerontol Geriatr 1991;22:53-59. 
-22 Wentzel C, Rockwood K, MacKnight C, Hachinski $\mathrm{V}$, et al: Progression of impairment in patients with vascular cognitive impairment without dementia. Neurology 2001;57:714716.

-23 Ingles JL, Wentzel C, Fisk JD, Rockwood K Neuropsychological predictors of incident dementia in patients with vascular cognitive impairment, without dementia. Stroke 2002;33: 1999-2002.

-24 Tian J, Bucks RS, Haworth J, Wilcock G: Neuropsychological prediction of conversion to dementia from questionable dementia: Statistically significant but not yet clinically useful. J Neurol Neurosurg Psychiatry 2003;74:433438.
25 Ballard C, Stephens S, McLaren A, Wesnes K, Kenny R: Mild cognitive impairment and vascular cognitive impairment in stroke patients. Int Psychogeriatr 2003; 15:123-126.

$\checkmark 26$ Frisoni GB, Galluzi S, Bresciani L, Zanetti O, Geroldi L: Mild cognitive impairment with subcortical vascular features: Clinical characteristics and outcome. J Neurol 2002;249: 1423-1432.

27 Rockwood K, Wentzel C, Hachinski V, Hogan DB, et al: Prevalence and outcomes of vascular cognitive impairment. Vascular Cognitive Impairment Investigators of the Canadian Study of Health and Aging. Neurology 2000;54:447451.
28 Tham W, Auchus AP, Thong M, Go ML, Chang HM, et al: Progression of cognitive impairment after stroke: One year results from a longitudinal study of Singaporean stroke patients. J Neurol Sci 2002;203-204(C):49-52.

29 Fisk JD, Merry HR, Rockwood K: Variations in case definition affect prevalence but not outcomes of mild cognitive impairment. Neurology 2003;61:1179-1184. 